Anuario Latinoamericano Ciencias Políticas

y Relaciones Internacionales

vol. 8, 2019

pp. 297-319

\section{El rol de la Unión Europea como actor externo en la política colombiana - entre el bilateralismo y el trilateralismo}

\author{
The Role of the European Union as an External Actor \\ in the Colombian Politics \\ - Between Bilateralism and Trilateralism
}

\author{
Klaus Bodemer* \\ INVESTIGADOR INDEPENDIENTE \\ HAMBURGO, ALEMANIA \\ $\triangle$ kbodemer@t-online.de \\ https://orcid.org/0000-0003-0101-1222
}

\title{
RESUMEN
}

Desde los años 90 del siglo pasado, la Unión Europea (UE) está percibida cada vez más como un actor global que dispone en su política exterior de una amplia variedad de instrumentos y actúa en varios niveles. Esta característica se manifiesta también en sus relaciones con América Latina, en general, y con Colombia, uno de los socios estratégicos en la región, en particular. El artículo hace un balance de las relaciones bilaterales estatales en las últimas dos décadas y media con el fin de precisar el rol que la UE está jugando como actor externo en la política colombiana. Desde el punto de vista teórico se utilizan planteamientos del interregionalismo institucional, del neorrealismo, de la perspectiva neoinstitucionalista y del constructivismo social. Para comprender la relación entre factores internos y externos, los conceptos del two-level game y de intermestic politics son dos aportes analíticos adicionales que nos permiten comprender mejor el objeto de nuestro estudio.

PALABRAS CLAVE: interregionalismo, relaciones europeo-latinoamericanas, relaciones UE-Colombia, trilateralismo, relaciones Sur-Sur.

\section{ABSTRACT}

Since the 90s of the last century, the European Union (EU) has been perceived more and more as a global actor that has a wide variety of instruments in its foreign

\footnotetext{
* Prof. Dr., Politólogo, exdirector del GIGA, Instituto de Estudios Latinoamericanos, Hamburgo.
} 
policy and acts at various levels. This characteristic is also manifested in its relations with Latin America, in general, and with Colombia, one of the strategic partners in the region, in particular. The article takes stock of state bilateral relations over the past two and a half decades in order to clarify the role that the EU is playing as an external actor in Colombian politics. From the theoretical point of view, approaches to institutional interregionalism, neo-realism, neo-institutionalist perspective and social constructivism are used. To understand the relationship between internal and external factors, we refer also to the analytical concepts of two-level game and intermestic politics.

KEYWORDS: interregionalism, Europe-Latin American relations, EU-Colombian relations, trilateral relations, South-South relations.

\section{Introducción: Las múltiples caras de la política exterior de la Unión Europea}

Desde la mitad de la década de 1990 observamos una expansión rápida de las áreas y la capacidad institucional de la política exterior europea. Esa política, basada en un sistema pluriactor y plurinivel, puede ser una oportunidad para implementar relaciones multifacéticas, pero también un riesgo, porque cada actor sigue sus propios intereses, percepciones y prioridades con el resultado de que las posiciones a veces coinciden, a veces se contradicen, los actores compiten, se bloquean mutuamente, no se toman mutuamente en cuenta o actúan en forma aislada. La complejidad descrita se encuentra también en las relaciones de la UE con Colombia, el caso que nos interesa aquí.

En lo que sigue quiero concentrarme en primer lugar en dos niveles de estas relaciones: el macro-nivel UE-CAN y el meso-nivel UE-Colombia. El micro-nivel, es decir, las relaciones entre Estados miembros de la UE con el país andino, puede ser tratado por falta de espacio solo puntualmente. Lo mismo vale para las relaciones entre actores no estatales (ONG y grupos económicos). Desde el punto de vista teórico, se utilizan planteamientos del interregionalismo institucionalizado y formalizado (proceso de interacción entre regiones a través de instituciones) (Doidge, 2011), del neorrealismo (relaciones en el contexto estatal) (Waltz, 1979), de la perspectiva neo-institucionalista (Keohane, 1984) y del constructivismo social (normas, valores, autopercepción, discurso) (Wendt, 1999). Para comprender la relación entre factores internos y externos nos han ayudado dos aportes analíticos adicionales, el de intermestic politics de Putnam (Putnam, 1988) y el de twolevel game de Pada (Pada, 2006). La interacción birregional entre actores no estatales, analizada con el concepto del transregionalismo (Soderbaum y Van Langenhove, 2006), debe quedar fuera de este trabajo por falta de lugar, a pesar de que estas relaciones han desempeñado un papel importante en las relaciones UE-Colombia. 


\section{La Comunidad Andina de Naciones (CAN) como socio de la Unión Europea: del discípulo modelo al enfermo crónico}

Dada su naturaleza híbrida y su objetivo de edificar un sistema multilateral efectivo, la UE ha sido un abogado "natural" de la cooperación e integración en otras regiones del mundo (Grevi, 2010, p. 11). La CAN, uno de los más viejos regímenes de integración en América Latina, hoy compuesto por Bolivia, Colombia, Ecuador y Perú, fue creada en 1969 como Pacto Andino por el Acuerdo de Cartagena y se ha orientado fuertemente al modelo europeo. Sin embargo, a diferencia del proceso de integración europea, la aplicación de los acuerdos ha sido insuficiente e incompleta debido a una serie de razones que van desde diferentes crisis en el ámbito internacional (las dos crisis de petróleo en la década de 1970, las deudas en la década de 1980), la debilidad político-institucional de los Estados miembros, conflictos entre ellos, cambios de la estrategia de desarrollo aplicada, la falta de incentivos para profundizar la integración, hasta el hecho de que sus economías no son complementarias. Tanto la denominación como los miembros y el rumbo estratégico de este régimen de integración cambiaron varias veces a lo largo del tiempo (Bodemer, 2000), lo cual ha afectado también sus relaciones exteriores.

Desde la firma del Acuerdo fundacional en 1969, la forma, la estrategia y las prioridades de las relaciones de la UE con la CAN han cambiado considerablemente. Esto es, en gran medida, un reflejo de las grandes transformaciones que se han dado en ambas regiones a lo largo de los años y de los efectos de la globalización económica. A eso se agrega la gran diferencia de poderes y de espacio internacional que la CAN y la UE tienen en el mundo y que determinan que la asimetría se mantenga como una característica fundamental de la relación euro-andina (Pacheco, 2002, p. 163).

El primer acuerdo birregional de relevancia entre la UE y la CAN se firmó en 1993 en Copenhague, denominado Acuerdo Marco de Cooperación. Este acuerdo, llamado de tercera generación, amplió el margen de maniobra con respecto del primer acuerdo no preferencial firmado en 1983. En 2003, este acuerdo fue ampliado en Roma por un diálogo político.

A pesar de los empujes que la relación UE-CAN ha experimentado en 2003-2004 por la agregación de un diálogo político, la crisis de la CAN en los años siguientes dificultó el proceso hacia una asociación. Negociaciones sobre un acuerdo de asociación se rompieron en el segundo semestre de 2008 por preferencias divergentes entre los miembros con respecto del libre comercio, lo cual resultó en un nuevo formato que ofreció una división temática y geográfica: continuas operaciones comerciales regionales con la CAN en su conjunto en el diálogo político y la cooperación, a la vez con un enfoque multipartes con aquellos países que estaban dispuestos a emprender negociaciones ambiciosas y amplias, compatibles con las reglas de la Organización Mundial del Comercio (modelo "OMC plus").
El rol de la Unión Europea como actor externo en la política colombiana - entre el bilateralismo y el trilateralismo

Klaus Bodemer 
Estas operaciones comerciales comenzaron en febrero de 2009 con Perú, Colombia y Ecuador. En julio 2009, Ecuador decidió suspender su participación. Solo Perú y Colombia estaban dispuestos a aceptar el formato de acuerdo de libre comercio "OMC plus" propuesto por la UE, mientras Bolivia y Ecuador lo rechazaron abiertamente, en particular sus capítulos sobre inversiones, propiedad intelectual y compras públicas, por su carácter "neoliberal" y sus efectos lesivos para el desarrollo. De ahí la decisión adoptada en enero de 2009 por el Consejo de la Unión Europea, por la que se abandonó la negociación interregional entre las dos regiones respecto del componente comercial, y más bien se acordó un mecanismo de flexibilidad para su negociación, por el que se determinó que cada Estado, "conforme a sus propias posibilidades, se incorporará en los pilares de dicho acuerdo y establecerá intensidades, plazos y velocidades" (Pastrana Buelvas, 2009, p. 30; Domínguez, 2013, p.106 y ss.). Como resultado, se negociaron acuerdos bilaterales de libre comercio con Colombia y Perú, aun manteniendo el marco interregional para el diálogo político y la cooperación al desarrollo (Sanahuja, 2013, p. 3). En la novena ronda de negociaciones realizada en Bruselas en marzo de 2010, Colombia y Perú concluyeron con la UE las negociaciones sobre los asuntos comerciales de un acuerdo de asociación. Los dos acuerdos de libre comercio entraron en vigor en 2013. Para Colombia, el tema más importante en las negociaciones ha sido el libre acceso de sus productos como banana, azúcar y tabaco al mercado europeo. Al no haber concretado un acuerdo de asociación con la CAN, la UE mantiene hoy acuerdos de libre comercio bilaterales multipartes con Colombia, Perú y Ecuador y un acuerdo de diálogo político y cooperación de tercera generación con todo el bloque y sus países miembros.

Tomando en cuenta las repetidas crisis del CAN y las idas y vueltas de sus relaciones con la UE, se puede decir que el peso de estas relaciones birregionales en las relaciones bilaterales UE-Colombia es más bien modesto. Las causas al respecto son diversas. Primero, para ninguna de las dos partes la otra es una prioridad. Un segundo elemento es la continua e inestable situación política en el espacio andino. Un tercer factor es el rol dominante de los Estados Unidos en la región y la controversia intraeuropea sobre la estrategia adecuada al respecto. Cuarto, no hubo constancia de membresía en la CAN, sino repetidas entradas y salidas. Quinto, el hecho de que compiten dentro de la región dos conceptos diferentes de democracia, de desarrollo e inserción internacional dificulta la formulación e implementación de estrategias comunes y un perfil claro hacia fuera. Finalmente, el modesto grado de interdependencia económica entre las dos regiones y la prioridad que la UE ha dado a otras regiones del mundo desde la caída del Muro de Berlín son factores adicionales que explican el rango subordinado de las relaciones birregionales UE-CAN (De Lombaerde et al., 2006, pp. 12-14). 


\section{Las relaciones Unión Europea-Colombia: el largo camino de un socio más bien periférico a un aliado político}

\section{El bajo perfil de las relaciones durante el gobierno de Álvaro Uribe (2002-2010)}

El rol de la Unión Europea como actor externo en la política colombiana - entre el bilateralismo y el trilateralismo

Klaus Bodemer

Las relaciones UE-Colombia son de larga data, pero no fue sino hasta la década de 1990, en el sistema internacional de la posguerra fría en el que su relación empezó a consolidarse (Friedrich Ebert Stiftung en Colombia, 2004, p. 1), sin ganar en los años siguientes, por otro lado, realmente una alta prioridad. A diferencia de los Estados Unidos, las razones económicas y estratégicas de un mayor compromiso de la UE jugaron más bien un rol subordinado y estaban limitadas a unos pocos miembros (sobre todo España). La posición europea se concentró, por tanto, por lo menos durante la primera década del siglo XXI, en rechazar el Plan Colombia, lanzado por el gobierno norteamericano. Este plan priorizó en su parte operativa la ayuda militar, lo que la mayoría de los países europeos rechazaron. Esta discrepancia no excluyó que, aparte de la Comisión Europea, el Parlamento Europeo y el Consejo Europeo emitieran repetidas veces sus opiniones acerca de la situación en Colombia, pero más allá de sancionar las declaraciones casi no hubo actos concretos (Kurtenbach, 2004, p. 54).

Frente a las estrategias divergentes entre la UE y los Estados Unidos con respecto a Colombia no sorprende que el perfil de la política exterior europea durante los años del gobierno Uribe (2002-2010), sobre todo durante su primer mandato (2002-2006), marcados por la estrategia de seguridad democrática, fuera poco coherente y su zona de aprovechamiento reducida. Eso, sin embargo, no ha excluido que la UE materializara su respaldo a la pacificación colombiana ya en 2000, es decir, durante el gobierno del antecesor de Uribe, Andrés Pastrana (1998-2002), y propusiera la creación de los llamados Laboratorios de Paz en Magdalena Medio, zona con un alto grado de violencia (el primero puesto en marcha en 2002, el segundo, en 2003), con ayuda a las reformas administrativojudiciales, la lucha contra la pobreza y los desplazamientos forzosos mediante un acuerdo básico de cooperación y asistencia técnico-económica con una dotación de $€ 105$ millones hasta 2006. La UE se distanció con los Laboratorios de Paz como poder blando de la estrategia militar de los Estados Unidos hacia Colombia y su instrumento, el Plan Colombia. Los Laboratorios de Paz siguieron un enfoque integral, con el propósito de atacar las causas del conflicto por liberar el potencial de desarrollo, reducir la intensidad del conflicto y los altos niveles de desigualdad (Pastrana y Aponte, 2006).

Los objetivos prioritarios de los Laboratorios de Paz fueron al nivel operativo los siguientes: primero, apoyar la realización de tratados específicos entre las partes en conflicto; segundo, crear una zona de convivencia pacífica de sus 
habitantes mediante el respaldo a instituciones locales y el apoyo de actores civiles que propagan la paz; y, tercero, empujar el desarrollo económico y social que fomente modelos de desarrollo alternativos con la cooperación de organizaciones de la sociedad civil y local. Ese tipo de proyectos correspondía por su concepto básico a lo que Mary Kaldor denomina "islas de civilidad" (Kurtenbach, 2004, p. 54 y ss.).

Las lecciones aprendidas de los Laboratorios de Paz durante los años 2002-2006 fueron mezcladas y sirvieron de base para el Documento de Programación Regional-América Latina 2007-2013 (Comisión Europea, 2007b), que por primera vez diseñó en detalle una estrategia integral de cooperación a mediano plazo. La UE tuvo éxito en crear un programa alternativo de paz desde abajo en medio de un conflicto armado, que incluyó vistas divergentes, pero engañó a sus socios, porque esta iniciativa fue demasiado débil para ser una verdadera alternativa a la estrategia oficial del Gobierno colombiano.

En cuanto a la asistencia técnico-económica, se constituyó el Grupo de Apoyo al Proceso de $\mathrm{Paz}$, que recomendaba como estrategia sin alternativa realizar negociaciones y adoptar políticas estructurales para mitigar las desigualdades, causas estructurales del conflicto. Sobre esta base se formó ya en 2001 una Mesa de Donantes o Grupo de los 24 (G24), que se encontró durante los años siguientes con cierta regularidad y en la que la comunidad internacional se comprometió a aportar 800 millones USD. La Comisión Europea reafirmó repetidas veces durante estos encuentros que no financiaría soluciones militares, condenó la fumigación de los cultivos ilegales, practicada por los estadounidenses, debido a sus efectos medioambientales desastrosos y el desplazamiento de personas. Los europeos abogaban, en cambio, por el fortalecimiento institucional, el diálogo, el desarrollo alternativo y la ayuda humanitaria, más acordes con el ideario europeo. También se puso en marcha el ECHO's Global Plan 2002 de acción humanitaria, financiado con otros 8 millones USD para quince meses (Delegación de la Unión Europea en Colombia, s/f).

Debido, en última instancia, al lobby del gobierno español, la UE cambió parcialmente en 2002 su posición respecto del conflicto colombiano e incluyó a las FARC (en 2004 también al ELN) en su lista de organizaciones terroristas sin abandonar por eso su interpretación de las causas estructurales del conflicto. En las conclusiones del Consejo de la Unión Europea sobre Colombia (2005), los Estados miembros expresaron su voluntad de colaborar con el gobierno legítimo de Colombia, pero subrayaron que esta colaboración estaría condicionada a la marcha del proceso de paz, a la implementación de una estrategia global de desmovilización, desarticulación, desarme y reinserción social de los grupos paramilitares y de satisfacción del derecho de las víctimas a saber la verdad y obtener justicia y reparación razonable.

A pesar de algunos nuevos acentos, las relaciones bilaterales se han mantenido durante la primera década del siglo XXI en un marco que da prioridad al compromiso por la solución pacífica negociada a un conflicto que tiene su causa, según la percepción europea, en una situación de pobreza y desigual- 
dad. Pero la implementación de esa estrategia fue obstaculizada, por lo menos durante el gobierno de Uribe (2002-2010), debido a dificultades para alcanzar consensos en el seno de la UE y la preferencia que algunos de los socios comunitarios han conferido a sus relaciones con los Estados Unidos y de sus formas de entender los problemas de Colombia. El Reino Unido, Italia, España (bajo el gobierno de Aznar) y algunos otros países miembros, como Holanda y Polonia, se han puesto de lado de la estrategia del gobierno de Bush, que, conforme a la interpretación del conflicto por el presidente Álvaro Uribe, ha defendido la tesis de que la respuesta a las luchas civiles y el conflicto armado, el cultivo, la elaboración y el tráfico de drogas debe ser prioritariamente militar, tomando en consideración que las FARC y el ELN son organizaciones terroristas que comprenden solo una respuesta militar. Expresión de esta percepción fue el Plan Colombia, que con su predominante enfoque (y presupuesto) militar fue recibido, sin embargo, por la mayoría de los países europeos y la Comisión Europea, como ya hemos mencionado, con mucha reserva.

\section{La descongelación y desecuritización de las relaciones mutuas durante el gobierno de Manuel Santos}

El cambio de gobierno de Álvaro Uribe a Juan Manuel Santos en 2010 ha facilitado un creciente compromiso de la UE con Colombia y una mayor receptividad del país andino a sus recomendaciones en temas como los derechos humanos y la sociedad civil (Duarte, 2012, p. 345). El nuevo presidente, exministro de defensa en el gobierno de Uribe, ha mostrado desde el comienzo de su mandato una cierta distancia frente a los Estados Unidos y un creciente interés por pertenecer a nuevos escenarios multilaterales. Esa estrategia incluyó terminar de ver el bloque europeo casi exclusivamente como una simple fuente de ayuda humanitaria y de cooperación al desarrollo en vez de un aliado político en muchos temas de la agenda internacional y promotor del deseo colombiano de formar parte de la Organización para la Cooperación y el Desarrollo Económicos (OCDE) (Arcos, 2015, p. 26).

Frente a la puesta en marcha del Tratado de Libre Comercio con los Estados Unidos y al hecho de que la UE fuera el segundo socio comercial y varios Estados miembros los principales proveedores de la inversión extranjera directa en el país andino, alcanzar un acuerdo comercial con la UE fue para el nuevo presidente un reto de primer orden y más conveniente para el país que el unilateralismo del SPG-Plus (Duarte, 2012, p. 345).

El tratado de libre comercio UE-Colombia fue ratificado en 2013, a pesar de una ola de protestas por parte de organizaciones de la sociedad civil que se quejaron sobre el deplorable estado de los derechos humanos en el país. Otro paso hacia una profundización en las relaciones bilaterales fue la búsqueda de apoyo técnico y financiero de la UE a la Ley de Víctimas y Restitución de Tierras que marcó una diferencia respecto del gobierno de Uribe.
El rol de la Unión Europea como actor externo en la política colombiana - entre el bilateralismo y el trilateralismo

Klaus Bodemer 
Con la "desecuritización" de la agenda exterior y los esfuerzos de la diplomacia de Santos para poner fin al autoaislamiento del país y su alineación casi exclusiva con su gran vecino del Norte, se abrió al país andino la oportunidad de insertarse activamente en la región, mejorar su posicionamiento internacional y colocar las relaciones con la UE y sus países miembros sobre una base más sólida.

Con la ampliación del enfoque de la política exterior colombiana bajo el gobierno de Santos, ambos lados tuvieron en cuenta que hay oportunidades de cooperación más allá del proceso de paz en el área de comercio y la cooperación al desarrollo. Con su creciente mercado y sus abundantes recursos naturales ${ }^{1}$, el país andino es para la UE un socio económico interesante. Con respecto a la cooperación al desarrollo, la UE ha promulgado ya en 2007 -paralelamente a la Estrategia de Cooperación Regional con la Comunidad Andina- una estrategia para Colombia que contiene un Plan de Acción para el periodo 2007-2013 (Comisión Europea, 2007a), que sigue la línea del Plan de Acción en el periodo anterior (2001-2006) y buscaba efectos a corto, mediano y largo plazo. Respecto al corto plazo, el propósito fue dar asistencia y apoyo a las víctimas de la violencia. A mediano plazo, la UE buscaba promover la paz local y nacional. Finalmente, aspiraba a contribuir a largo plazo a una resolución duradera del conflicto armado mediante la promoción de un mejor desarrollo para todos, que tomara en cuenta las raíces estructurales del conflicto. Como primer donante, la UE privilegió, según la Estrategia País, tres ejes básicos de cooperación con un presupuesto de $€ 160$ millones: primero, paz y estabilidad, incluido el desarrollo alternativo (70 \% de los fondos); segundo, Estado de derecho, justicia y derechos humanos (20\% de los fondos); y, tercero, el $10 \%$ restante para temas de productividad, competitividad y comercio. Más allá de estos tres ejes temáticos, la protección de la biodiversidad y del medio ambiente fue un cuarto eje con creciente importancia para la UE. De 2015 en adelante, el Country Strategy Paper fue sustituido por el Programa Indicativo Multianual como nuevo marco de programación basado en el alineamiento con las políticas y los planes nacionales de desarrollo (Sanahuja, Tezanos, Kern y Perrotta, 2015, p. 38).

\section{La membresía de Colombia en los nuevos regímenes de integración: ¿factor impulsor de las relaciones mutuas?}

Una variable adicional que influye en las relaciones entre e la UE y Colombia es su membresía en la Unasur, la CELAC, y - desde reciente - en la Alianza del Pacífico (AP). La percepción europea de estos nuevos regímenes de integración

1 Colombia dispone de una gran riqueza de petróleo, minerales metálicos (hierro, oro, níquel, cobre, plata, platino), no metálicos (sal terrestre, sal marina, gravas, arenas, arcilla, azufre, bentonita, asbesto, magnesita, talgo, yeso, entre otros) y piedras preciosas (esmeraldas). 
no fue clara como tampoco la cuestión de cómo los europeos deberían reaccionar a ellos. La Unasur y la CELAC provocaron en Bruselas, por un lado, ciertas expectativas, porque son ejemplos de un nuevo empuje para el regionalismo sudamericano que la UE siempre ha favorecido; se critica, por otro lado, la manía latinoamericana de crear cada vez nuevos regímenes de integración en vez de hacer lo máximo posible para que los ya existentes funcionen. En general, Colombia es vista desde Europa como económicamente fuerte secondary power, como un factor de estabilidad en la subregión andina, comparable a Brasil como sub-hegemón en el Cono Sur. Colombia dispone, además, de una economía fuerte, es rica en recursos naturales de los cuales Europa carece y, además, con la conclusión exitosa del proceso de paz en 2016, es vista desde Europa como palanca político-ideológica contra su país vecino, el troubleshooter permanente Venezuela. Colombia se ha transformado bajo el gobierno de Santos sucesivamente en el secondary power más fuerte en América Latina (Flemes y Castro, 2015 , p. 204). Además, este país comparte con los europeos la convicción de que la democracia representativa, el regionalismo abierto, el libre comercio y una inserción activa en el mercado mundial son los mejores fundamentos para un desarrollo duradero. Como miembro de la Alianza del Pacífico, el esquema de integración más joven en la región, Colombia constituye finalmente, junto con Perú, México y Chile, la fracción liberal en América Latina, que aboga por una relación pragmática y desideologizada con el hegemón del Norte, los Estados Unidos. El país andino se distancia con eso de Ecuador y Bolivia que, junto con Venezuela, median por un desarrollo más autónomo, una democracia directa, inclusiva y popular y una economía más cerrada.

Mientras el Mercosur y la AP son ejemplos del "regionalismo abierto" (si bien el primero ha violado esta estrategia en la práctica a veces con medidas defensivas y proteccionistas), la Unasur es más bien un ejemplo de integración pos-neo-liberal y la CAN dividida entre una fracción liberal (Perú y Colombia) y una que oscila entre el modelo posliberal y la Alianza Bolivariana para los Pueblos de Nuestra América (con Ecuador y Bolivia) y Venezuela que persigue bajo el lema del socialismo del siglo XXI un concepto ideológicamente muy difuso, una estrategia nacional-populista con un componente antiliberal y antiestadounidense fuerte (Welsch y Briceño, 2011). Otro fenómeno que habla, visto desde la UE, en favor de la AP y sus países miembros es el hecho de que esta Alianza está prácticamente integrada en el Acuerdo Transpacífico de Cooperación Económica. Tres de los cuatro países que lo integran -México, Chile y Perú - forman parte de las negociaciones. Se encuentran trece países miembros de la UE entre los países observadores de la AP. Su conclusión podría añadir dificultades para vertebrar un mercado integrado en la región. En particular Brasil está en riesgo de ser aislado, un motivo más para que este país esté buscando fórmulas con el propósito de revitalizar un acuerdo con la UE, con sus socios del Mercosur o sin ellos (Steinberg, 2014).

Con la CELAC, que es en primer lugar un instrumento de coordinación política, la UE tiene por primera vez un verdadero interlocutor. Queda, sin em-
El rol de la Unión Europea como actor externo en la política colombiana - entre el bilateralismo y el trilateralismo

Klaus Bodemer 
bargo, una pregunta abierta si este mecanismo puede cumplir realmente el rol de un interlocutor biregional coherente en los años venideros como se espera en Bruselas. La Unasur es, finalmente, un nuevo régimen de cooperación económica intrarregional, concentrado en la promoción de ciertas políticas sectoriales (infraestructura, etc.). Si este régimen de integración posliberal produce, más allá de la retórica de sus protagonistas, resultados concretos frente a las crecientes dificultades económicas en varios de sus países miembros (Loser, 2015), no está garantizado. La UE parece compartir este escepticismo, como una entrevista del autor con el delegado de la UE para Colombia y Ecuador en Bogotá el 25 de febrero de 2015 ha revelada. Si, por otro lado, las economías de la Unasur se recuperan otra vez, sin duda se ofrecerá a la UE una serie de oportunidades para estrechar las relaciones mutuas, lo cual podría tener el efecto colateral y más bien indirecto de fortalecer también las relaciones UE-Colombia.

\section{Factores limitantes de una relación más sustancial entre la Unión Europea y Colombia}

Los intentos de un fortalecimiento de las relaciones UE-Colombia se ven enfrentados, más allá de los efectos del complejo sistema de la política exterior, de una serie de cuestiones internas limitantes en ambos lados.

\section{Factores limitantes en Europa}

Son en primer lugar siete factores que tienen un efecto limitante respecto las relaciones birregionales entre la UE y América Latina en general, y las relaciones con Colombia en particular:

(1) La UE está sufriendo en los últimos años una crisis múltiple que hace erosionar su imagen, la cuestiona como modelo de integración, poder civil (soft power) y Estado de bienestar. Los fenómenos más visibles al respecto son la crisis del euro como consecuencia de la crisis financiera internacional (2008/2009) y los errores en la introducción de la moneda única. Observamos, además, una brecha creciente de desarrollo tanto dentro de la UE como entre el norte y el sur de esta, lo cual circunscribe un conflicto entre los principios/ objetivos de la competitividad (según el Tratado de Lisboa), propagado por sus países más consolidados, y el de solidaridad, anclados ya en los tratados fundadores y reclamados en primer lugar por los países sobreendeudados, en su mayoría en el sur de Europa. El caso ejemplar es la difícil relación entre la UE y Grecia.

(2) España, durante décadas uno de los protagonistas en las relaciones europeo-sudamericanas, ha perdido como consecuencia de la crisis económica (recesión fuerte) y una crisis política (escándalos de corrupción) este rol en los años recientes. 
(3) Más allá de los graves problemas económico-financieros, la UE sufre bajo una crisis de legitimidad, reflejada en el auge de movimientos y partidos claramente antieuropeos o por lo menos anti-Bruselas. Sus ingredientes son el rechazo de la democracia liberal representativa en favor de prácticas de democracia directa. Estas nuevas articulaciones políticas van más allá de la separación derecha-izquierda, se reclutan tanto de la clase media como de clase media-baja y baja. Sus adherentes prefieren un discurso populista y nacionalista y son en parte contaminados por la xenofobia, el odio de razas y el antisemitismo. Objeto de su rechazo son inmigrantes, refugiados y otras minorías, pero también la clase dirigente, "aquellos de arriba", que, según su crítica, se han alejado de los ciudadanos y sus preocupaciones cotidianas.

(4) Como consecuencia de estos fenómenos de crisis mencionados, observamos en Europa una política de ensimismamiento, una mirada hacia dentro, acompañada por el auge del nacionalismo (por ejemplo, en Hungría, Polonia y Eslovaquia). Emblemática es al respecto la crisis económico-financiera en la Europa mediterránea y la reacción de algunos gobiernos europeos a la nueva ola de migraciones. El Acuerdo de Schengen no existe más.

(5) Mirando específicamente a las relaciones europeo-sudamericanas: estas nunca fueron una prioridad en la política exterior europea, tampoco durante los años dorados de la década de 1990 y el periodo de las "vacas gordas" (2003-2008) gracias al boom de los altos precios de los recursos naturales. El foco de interés de la UE se ha dirigido durante las últimas décadas cada vez más a Asia, sobre todo China e India (por motivos económicos y políticos), África (como objeto principal de la cooperación al desarrollo), Oriente Medio (por las turbulencias políticas y el terrorismo islámico) y Europa del Este, es decir, los nuevos miembros de la UE y sus vecinos, incluidos los países balcánicos (con la llamada política de vecindad como respuesta). Tanto la atención política como los recursos financieros y de personal son, por tanto, con respecto a América Latina y el Caribe más bien limitados y experimentaron en el nuevo presupuesto de la UE 2014-2020 recortes adicionales.

(6) Si nos preguntamos cuáles son los países en Europa que tienen un interés verdadero en América Latina, son, de los 28 países, no más que un tercio, en su mayoría de Europa del oeste y norte, y de ellos quedan como resultado de la crisis económica en Europa finalmente no más que cuatro: Alemania, Francia, España y Holanda, y, si bien menos pronunciados, Italia, Suecia y Inglaterra.

(7) Otros factores limitantes tenían que ver con la percepción europea de los cambios políticos de 2003 en adelante en América Latina, es decir, la vuelta a la izquierda y sus reflejos en el rumbo de sus políticas exteriores. Las reacciones europeas a estos cambios fueron poco coherentes y han nutrido en América Latina la sospecha de que existe en Bruselas una cierta desorientación, sobre todo en cuanto a los gobiernos nacional-populistas en Venezuela, Bolivia y Ecuador con sus nuevos líderes políticos, cuyos estilos de gobierno y fuentes de poder difieren de aquellos de las élites tradicionales (constitucio-
El rol de la Unión Europea como actor externo en la política colombiana - entre el bilateralismo y el trilateralismo

Klaus Bodemer 
nes fundacionales en Ecuador, Venezuela y Bolivia, nuevas articulaciones de democracia directa, inclusión de capas de la población hasta ahora marginalizadas en el proceso político). Otra fuente de cierta desorientación en Europa son, como ya hemos mencionado, los nuevos regímenes de integración, como la Alianza Bolivariana para los Pueblos de Nuestra América (ALBA), Unasur y la CELAC y su compatibilidad con los regímenes de integración de vieja data (CAN, Mercosur).

\section{Factores limitantes en América Latina en general y Colombia en particular}

Factores de freno de una relación bilateral más ágil son al lado latinoamericano:

(1) La pérdida de atracción de Europa: Como reacción a los factores de crisis en Europa, su imagen como región de paz, modelo de integración, de democracia y bienestar ha sufrido mucho en años recientes en América Latina. Hoy Europa aparece ante muchos latinoamericanos más bien como foco de problemas que de solución. Uno de los reproches es que Europa está repitiendo los errores de las políticas de ajuste que en América Latina fueron aplicadas como reacción a la década pérdida y está forzando a países como Grecia, Irlanda, España, Chipre y Portugal a aplicarlos sin más. Otra queja es que los europeos no han reconocido suficientemente los avances económico-sociales de las economías sudamericanas, implementados por los nuevos gobiernos de centroizquierda e izquierda durante los "años dorados" (2003-2010), basados en reformas estructurales profundas que les han permitido sobrevivir a las turbulencias financieras internacionales mucho mejor que los países de la zona euro y Estados Unidos.

(2) La sensación de que la UE sigue aferrándose con respecto a sus relaciones birregionales en las categorías Norte-Sur y no toma en cuenta que hay una creciente autoestima de las élites sudamericanas. Esta creciente autoestima de las élites sudamericanas se refleja también en su política exterior y se expresa en la demanda hacia el Norte por más simetría y la aceptación del hecho de que el peso de las relaciones Norte-Sur tradicionales está disminuyendo y que se abren a los países del Sur nuevas opciones de cooperación Sur-Sur y triangular (Morazán, Sanahuja y Ayllón, 2011, p. 15 y ss.; Bancet, 2012; Dominguez, 2017).

(3) Con respecto a Colombia, hay fuertes indicios de que hay en tiempos recientes una serie de factores que dificultan el aprovechamiento pleno de las nuevas oportunidades en su política exterior. Hay en particular dos factores de freno. El primero tiene que ver con la resonancia regional que ha encontrado el anuncio del gobierno de Santos de que el país podría firmar un acuerdo con la Organización del Tratado del Atlántico Norte (OTAN). Este anuncio ha desatado en el entorno latinoamericano un vendaval de declaraciones en 
primer lugar en países vinculados con la ALBA. ${ }^{2}$ El anuncio de Santos es balanceado, por otro lado, por la participación del país andino - después de una primera fase de reticencia- en el Consejo de Defensa Suramericano, impulsado por el gobierno de Lula da Silva. A pesar de este paso de acercamiento del gobierno colombiano a sus vecinos del Sur, en primer lugar, Brasil, los enfoques para la defensa y seguridad de ambos países siguen siendo muy distintos (Pastrana y Vera, 2015, p. 187). Un segundo factor, contrario a un rol más protagónico de Colombia en el ámbito regional e internacional, es el efecto de la caída del precio de petróleo a los ingresos fiscales de este país. Estos ingresos disminuyeron significadamente a causa de la caída de los precios de las materias primas. La renta petrolera fue en 2015 un $60 \%$ menor que la de 2013. Las exportaciones mineras disminuyeron considerablemente la entrada de divisas. Las medidas adoptadas por el gobierno para mitigar la crisis fueron más bien paliativas y dolorosas para la población, pero no señalan un cambio de paradigma, que sería necesario. La bonanza de los años pasados no fue aprovechada bien por el gobierno colombiano, sobre todo con respecto al petróleo. Si bien el ingreso de divisas alimentó el gasto público, no se corrigieron los problemas más graves: los cuellos de botella en infraestructura y la regulación que ha retrasado la exploración para aumentar las reservas. El sector petrolero del país es, además, no tan atractivo para invertir, porque, más allá de la deficiente infraestructura, las reservas de petróleo son relativamente pequeñas y los costos de explotación más altos que los de sus competidores México, Venezuela y Brasil (Cala, 2015).

\section{Factores que ayudan a fomentar las relaciones entre Europa y Colombia}

Existen, sin embargo, también elementos que hablan en favor de una profundización de las relaciones europeas con Colombia. Entre ellos merecen ser mencionados los siguientes:

(1) Ambas partes comparten los valores occidentales: democracia representativa, derechos humanos, solución pacífica y negociada de conflictos, soberanía y no injerencia en asuntos internos, economía social de mercado, multilateralismo, desarrollo sostenible y gobernanza global. Este consenso con respecto a ciertos objetivos no excluye, sin embargo, que las interpretaciones de tales valores o el posicionamiento de cada valor en la jerarquía de valores a veces discrepen entre ambas partes. Hay ciertas diferencias entre Bru-

\footnotetext{
2 Un ejemplo es una declaración del ministro boliviano de defensa, Rubén Saavedra, quien señaló: "Cualquier forma de presencia de la OTAN en Sudamérica o Latinoamérica ya implica un riesgo para la paz en la región. Esta presencia también implica desestabilización de la misma región, un riesgo inminente de la paz que gozamos" (citado por Malamud y García, 2013, p. 1). Otra voz crítica vino del presidente venezolano, Nicolás Maduro.
}

El rol de la Unión Europea como actor externo en la política colombiana - entre el bilateralismo y el trilateralismo

Klaus Bodemer 
selas y los países latinoamericanos (y entre ellos Colombia), por ejemplo, con respecto a las connotaciones que se vinculan con el término multilateralismo efectivo. Lo mismo vale para el término soberanía. Colombia es como los otros países latinoamericanos reticente a la implementación de mecanismos supranacionales, expresión del alto valor que se atribuye al principio de soberanía en esta región. Estas discrepancias son, sin embargo, más bien de matices y no excluyen relaciones mutuas fluidas.

(2) El hecho de que todos los actores involucrados en las relaciones bilaterales están incluidos en procesos de integración, que son variantes del regionalismo abierto y postliberal y buscan combinar competitividad con solidaridad, bienestar social con crecimiento económico, si bien con acentos que varían, es sin duda un factor positivo.

(3) Colombia es como Brasil un socio prioritario y estratégico de la UE en la subregión. Este fenómeno y el respaldo de la UE a los nuevos regímenes de integración, Unasur y la CELAC, subrayan que la UE no ha abandonado su vieja estrategia del birregionalismo, persigue más bien cada vez más relaciones de "geometría variable". Bruselas ha declarada además en varias oportunidades su interés en una cooperación más estrecha entre el país andino y Brasil y en el éxito de la AP como un nuevo tipo de integración transregional, que encuentra su correlato en el Acuerdo Transpacífico de Cooperación Económica y el Tratado Transatlántico de Comercio e Inversiones, que siguen la misma lógica del regionalismo abierto. El hecho de que los miembros de la AP sean aquellas economías que disponen de los mejores indicadores macroeconómicos en América Latina está visto en Bruselas como un factor adicional de esperanza frente al estancamiento o retroceso económico en los otros regímenes de integración en América Latina.

(4) Como reacción al fracaso del Área de Libre Comercio de las Américas (ALCA) en la Cumbre Hemisférica en Mar del Plata en diciembre 2005, el gobierno estadounidense se ha despedido de su (solo pasajero) respaldo para un enfoque regionalista y regresó a su vieja estrategia de hubs and spokes por medio de una serie de tratados de libre comercio (con Chile, los países centroamericanos, Perú y Colombia). La UE, por su parte, respondió a esto con una estrategia similar, relativizó su estrategia hasta ahora privilegiada de negaciones región a región y la completó con tratados bilaterales. Tanto el Acuerdo de Asociación Estratégica con Brasil (2007) como los acuerdos de libre comercio con Perú, Colombia (2013) y Ecuador en 2016 pueden ser comprendidos en este contexto como intentos de "abre puertas": en la CAN para incentivar al restante país de este régimen, Bolivia, a saltar al tren y firmar un tratado de libre comercio con la UE; en Brasil para desbloquear, con este socio privilegiado, las negociaciones UE-Mercosur. No está previsto en esta línea estratégica de la UE la idea de que Colombia como secondary power deba ser comprendida y fomentada por la UE como una potencia que responde a posibles ambiciones hegemónicas de Brasil, sino más bien como un país cooperante que, junto con su vecino Brasil, aporta a fomentar la estabilidad 
regional y robustece el campo de aquellos países en el subcontinente que tienen la mayor afinidad normativa con el viejo continente y refuerza su poder de negociación en la arena internacional. Finalmente, a pesar del auge del bilateralismo en las relaciones europeo-sudamericanas, este no ha reemplazado el birregionalismo, sino que ambas estrategias deben más bien ser vistas como complementarias (Bodemer, 2015, p. 393).

\section{Primeros pasos hacia una relación trilateral UE-Colombia-Brasil}

Como ya hemos mencionado, la UE percibe tanto Colombia como Brasil como poderes de estabilidad en sus subregiones y por eso como socios prioritarios y estratégicos en América Latina. Una consecuencia de eso es que Bruselas está cada vez más interesado en una relación trilateral (UE-Colombia-Brasil), lo que presupone como base una relación más estrecha entre los dos países latinoamericanos. ¿Es eso una perspectiva realista?

Una política proactiva de la UE hacia una relación cooperativa entre Colombia y Brasil presupone que los actores relevantes de ambos países están realmente interesados en esta- y son capaces de implementarla. Según pronunciamientos de los viejos y nuevos gobiernos en ambos países (Manuel Santos y Iván Duque en Colombia; Lula da Silva, Dilma Rousseff, Michael Temer y Jair Bolsonaro en Brasil), este interés existe realmente y se ha transformado ya antes de la firma del acuerdo de paz entre el gobierno y las FARC a finales de 2016 en una serie de proyectos de cooperación concretos entre los dos países latinoamericanos. Este compromiso bilateral es un fenómeno relativamente nuevo, porque el país andino ha tenido tradicionalmente, y a diferencia de Chile y Argentina, una posición más bien distante a su vecino Brasil, resultado de su estrecha relación (bandwagon) con los Estados Unidos y la preeminencia de intereses de seguridad. La relación estrecha con el poder hegemónico del Norte fue reforzada bajo el gobierno de Uribe y fue vista por los países vecinos, entre ellos Brasil, con gran escepticismo. Uribe creía, por otro lado, que Brasil estaba apoyando a las FARC y fue reticente a una Unasur liderada por Brasil, porque recelaba de que este nuevo régimen de integración podría ser utilizado por sus miembros como plataforma de un crudo antiamericanismo y rechazo del Plan Colombia (Flemes y Wehner, 2015, p. 9; Cardona Cardona, 2011). Con el cambio de rumbo de la política exterior bajo las dos administraciones de Santos (de 2010 hasta 2018), esta fijación de la política exterior colombiana en el tema de seguridad y el privilegio que han gozado los Estados Unidos en la política exterior colombiana terminó. Una nueva época de vecindad Colombia - Brasil fue iniciada por el Primer Investment Forum Colombia-Brasil en octubre de 2011, donde los dos mandatarios, refiriéndose a un informe del Banco Interamericano de Desarrollo (BID) ("Breaking down the Wall”), anunciaron una nueva cooperación entre ambos países, basada en proyectos ambiciosos de infraestructura y de expansión
El rol de la Unión Europea como actor externo en la política colombiana - entre el bilateralismo y el trilateralismo

Klaus Bodemer 
del comercio (Zibechi, 2011). Santos daba margen para una amplia gama de relaciones comerciales y de seguridad con los países vecinos de América del Sur con énfasis en Brasil y, más allá de la región, con países de la Cuenca del Pacífico. Ambas estrategias parciales sirvieron a Colombia para aumentar su capacidad de soft balancing y buffering frente a Brasil y subrayar su ambición de "liderazgo de segunda fila" (Flemes y Wehner, 2015, p. 10). Finalmente, la caótica situación venezolana ha subrayado aún más para la UE el peso tanto de Colombia como de Brasil en la región y la importancia de un fortalecimiento de las relaciones bi- y trilaterales.

\section{Balance y perspectivas de las relaciones Unión Europea - Colombia después del tratado de paz con las FARC}

(1) Colombia es hoy el socio más importante y confiable de la UE en la región andina. Las relaciones mutuas se desarrollaron constantemente en los años recientes. Desde comienzos de 2012, la UE ha apoyado además como "poder blando" las negociaciones de paz entre el gobierno colombiano y las FARC en La Habana y ha subrayado su disponibilidad de contribuir económicamente a la implementación de un acuerdo. Las negociaciones fueron concluidas exitosamente el 23 de noviembre de 2016 con la firma del Acuerdo de Paz por ambas partes de negociación y aprobado poco después por el congreso colombiano. Los países garantes y acompañantes - la UE, Noruega, Alemania (entre otros) - han cumplido un papel decisivo en el proceso de paz, en el caso de la UE y Estados Unidos también a través de sus enviados especiales. La contribución europea consistía en tres áreas: apoyo en la mesa de negociación, apoyo financiero y apoyo político. Este apoyo fue también mantenido en la fase posconflicto. En mayo de 2017, la UE anunció fondos de un paquete de casi 600 millones de euros (unos 639 millones de dólares), que incluye préstamos del Banco Europeo de Inversiones (BEI) de 400 millones de euro, así como subvenciones a través de un fondo fiduciario por un monto de 95 millones de euros. Estos fondos se destinan principalmente a tareas de desarrollo rural, justicia y desminado, entre otros, y van a ser integrados en el Plan Marco que articula las labores del Gobierno Nacional con los departamentos y municipios.

(2) Más importante que el aporte financiero de la UE y de algunos de sus países miembros fue su aporte político. Mientras que la participación de Venezuela y Cuba en las negociaciones de paz entre el gobierno y las FARC fue fuertemente criticado por la oposición colombiana, la de la UE a través del irlandés Eamon Gilmore (como enviado especial), gran conocedor del proceso de paz en Irlanda del Norte y de Noruega como país garante fue bienvenido. Finalmente, la eliminación de las FARC del listado de organizaciones terroristas de la UE fue un gesto político europeo significativo que mostró que la paz avanzaba también en el espacio internacional (Johnson, 2017). 
(3) Tras la entrega de las armas por parte de las FARC, la UE ha pronunciado a través de su Representante en Bogotá, la portuguesa Ana Paula Zacarís, que quiere trabajar en tres áreas: primero, la reincorporación de las ex-FARC en la sociedad y la vida civil y política; segundo, el acompañamiento internacional para el desarrollo rural integral; y, tercero, el apoyo para la unidad especial de la fiscalía para la lucha contra el crimen organizado (Sin autor, 2017).

(4) Con el acuerdo de paz entre el gobierno y las FARC se vinculó la esperanza de que el conflicto armado de más de cincuenta años de duración que ha costado 220000 muertos y 6 millones de desplazados, cifra que, según datos de las Naciones Unidas, solo es mayor en Siria, haya llegado a su fin. Al mismo tiempo fue argumentado que la implementación de los resultados del proceso de paz exige, si las soluciones deben ser duraderas, una fuerte participación internacional (Maihold y Naucke, 2015, p. 3). Se recomendó, por ejemplo, aprovechar la competencia de expertos internacionales para la implementación de la llamada justicia transicional. Un ejemplo al respecto es Alemania que presta una ayuda importante para una paz duradera con el proyecto ProFis de la Deutsche Gesellschaft für Internationale Zusammenarbeit, GIZ (Agencia Alemana de Cooperación Internacional). Organizaciones internacionales, como las Naciones Unidas, el Banco Mundial, la OCDE, la OEA y la UE están desempeñando también un papel importante en el proceso posconflicto y apoyan y acompañan el proceso de paz con una amplia gama de medidas.

(5) Hubo desde el comienzo de la fase postconflicto dudas justificadas sobre la capacidad del Estado colombiano de estar presente en todo el país y llenar el vacío de poder que la desmovilización de las fuerzas ilegales ha dejado en amplias zonas. Como punto de referencia al respecto sirvió el ejemplo del posconflicto en Centroamérica, donde la violencia después de la firma de los tratados de paz de ningún modo terminó. Hecho es en el caso de Colombia que, una vez logrado el desarme de las FARC, el ELN, el segundo grupo rebelde más importante del país, y bandas criminales como el Clan del Golfo son los principales escollos para la pacificación duradera del país. Si bien el ELN mantiene desde febrero de 2017 diálogos de paz con el gobierno - al comienzo con Santos, desde las elecciones en junio 2018 con su sucesor Iván Duque - este proceso fue interrumpido varias veces con el resultado que la ELN sigue siendo hasta la actualidad (el comienzo de 2019) uno de aquellos actores que más violencia genera. Con unos 1500 combatientes y varios miles de milicianos, repartidos según el gobierno en una docena de departamentos, el ELN reivindica el secuestro, la extorsión, los atentados a infraestructuras y oleoductos como parte de la lucha insurgente.

(6) Un desafío adicional que agrava el conflicto social en Colombia también en los tiempos post-acuerdo con las FARC es la pugna por el control de la coca, una lucha, en la cual están involucrados tanto disidentes de las FARC como la policía, el ejército, hasta bandas criminales. Como reacción a protestas recientes de cocaleros y una serie de enfrentamientos violentos
El rol de la Unión Europea como actor externo en la política colombiana - entre el bilateralismo y el trilateralismo

Klaus Bodemer 
con las fuerzas de seguridad, el gobierno ha reaccionado con la militarización del Sur del país (Manetto, 2017). La desmovilización de los paramilitares en 2002-2004 ha mostrado además que el $20 \%$ de los miembros de las bacrim se han constituido de nuevo y quedaron activas sin que las fuerzas policiales regulares fueran hasta ahora capaces de frenarlas. Ya se habla, por tanto, de un posible problema "facrim" (Maihold y Naucke, 2015, p. 5).

(7) En marzo de 2018, los colombianos eligieron un nuevo parlamento, en mayo y junio en dos rondas un nuevo presidente. Frente al hecho de que ningún candidato alcanzó una necesaria mayoría en la primera ronda, en la segunda ronda ganó, como ya en las elecciones parlamentarias, la fracción de derecha con Iván Buque con el expresidente Uribe en el fondo. Con esta victoria de la derecha creció el peligro real que el acuerdo de paz no vaya a ser implementado en gran parte, aún menos que se vaya a iniciar una reforma agraria que da a los pequeños campesinos más derechos. Nadie supo cómo las FARC reaccionarían a eso. Según un informe de la Fundación Paz y Reconciliación, la cual ha hecho un balance de lo alcanzado en un año de paz, hasta el momento el congreso ha implementado solo 8 de las 24 reformas de ley. El cultivo de coca creció, la construcción de rutas y otros objetos de infraestructura sigue siendo embrionario y 1000 miembros de las FARC siguen siendo encarcelados, a pesar de la ley de amnistía. Más que 700 guerrilleros siguen luchando en diferentes grupos. En 70 comunidades, antes en manos de las FARC, hubo una irrupción de una nueva violencia que es más fuerte que antes. Cada cuatro días se ha asesinado un activista de los movimientos sociales (Fundación Paz \& Reconciliación, 2017).

(8) Frente a la desconfianza entre las FARC y los militares, una misión cívica internacional ha vigilado el proceso de desmovilización, también más allá del desarme. Gracias al prestigio moral que ha ganado la UE con sus Laboratorios de Paz, su participación en este proceso fue bienvenida, así como el apoyo de algunos países miembros de la UE, por ejemplo, Alemania que dispone de experiencias en este terreno. Existían al respecto también experiencias de la Misión de Apoyo al Proceso de Paz en Colombia de la Organización de los Estados Americanos, la OEA, con el apoyo financiero del Bundesministerium für Zusammenarbeit und wirtschaftliche Entwicklung, BMZ (Ministerio Federal para la Cooperación y el Desarrollo Económico), que ya ha acompañado la desmovilización de los paramilitares años atrás (Maihold y Naucke, 2015, p. 4). Al comienzo se había pensado también en un apoyo por parte de la Unasur; sin embargo, esta institución prácticamente no puede actuar más desde el retiro reciente de algunos de sus países miembros que lamentaron la politización e instrumentalización de este organismo por el régimen chavista y sus aliados. Además, Unasur no dispone de momento ni de experiencias ni de la capacidad financiera y o de personal para comprometerse substancialmente al respecto.

(9) Un compromiso más fuerte de la UE en Colombia es de esperar de la llamada cooperación triangular dentro de la región y más allá de sus fronteras (Sotillo y Bendelac; Morazán, Sanahuja y Ayllón, 2013; Duarte, 2014, pp. 
450-452; Bancet, 2012). El cambio de paradigma de la UE en su cooperación al desarrollo ha abierto (y hecho necesario) nuevos caminos al respecto. La progresiva retirada de la ayuda europea de aquellos países latinoamericanos con una mayor renta per cápita, entre ellos Colombia, como se manifestó en el Marco Financiero Plurianual 2014-2020, y la consecuente concentración de los fondos europeos de cooperación en los países más pobres, fueron motivos para explorar la emergente cooperación Sur-Sur en proyectos triangulares Norte (UE)-Sur (por ejemplo, Colombia) con otros socios en América Latina y más allá de la región. El contexto regional parce facilitar una cooperación de este tipo. Las acciones de cooperación Sur-Sur de Colombia bajo la presidencia de Santos estuvieron orientadas a la cuenca del Caribe (Programa Regional de Cooperación con Mesoamérica), Asia y África (Agencia Presidencial de Cooperación Internacional de Colombia 2015). Con respecto a la cooperación triangular de la UE, Colombia se queda entre los países socios hasta ahora más bien al margen, pero existen oportunidades para ampliarlo, por ejemplo, en el área de las pymes, el desarrollo agrario, la ciencia y la tecnología, el medio ambiente, el desarrollo local y la lucha contra el crimen organizado transnacional.

(10) A fin de dar continuidad a los procesos que la UE ha desarrollado en Colombia, ambas partes han decidido cooperar con prioridad en dos líneas de acción: por un lado, en el área de la cohesión territorial; y por el otro, en el campo del comercio sostenible. Además, el gobierno de Colombia ha manifestado en las negociaciones con la UE su interés de acceder a nuevos instrumentos de financiación que tradicionalmente han sido poco utilizados, como los mecanismos de cooperación regional y de temáticas coordinadas desde Bruselas. Para esto, se han explorado las diferentes vías con posibilidad de financiación de proyectos en Colombia con convocatorias nacionales o globales (Agencia Presidencial de Cooperación Internacional de Colombia, 2015).

(11) Con respecto a posibles aportes de los Estados miembros de la UE, se ha divulgado que Francia y Alemania han prometido una ayuda concreta. En Francia se trata de apoyo para un programa de formación técnica con el objetivo de mejorar las perspectivas de los jóvenes en el campo y de calificar a la policía en el campo según el modelo de la gendarmería francesa e incorporar excombatientes de las FARC (Maihold y Naucke, 2015, p. 5). El compromiso de Alemania incluye no solo el gobierno y varios ONG, sino también el Parlamento Alemán que ha publicado en 2017 una moción, compartida por los grupos parlamentarios CDU/CSU, SPD y Alianza 90/Los Verdes que subraya su acompañamiento a lo que resta del proceso de paz con las FARC y a los procesos con la ELN (Ambos, 2016). Este apoyo se ha intensificado con la designación de Tom Koenigs, diputado del Bundestag, como enviado especial del Ministerio de Relaciones Exteriores para el proceso de paz. En el ámbito financiero, el Gobierno alemán desembolsó a través del Banco Alemán de Desarrollo 75 millones de euros (unos 78 millones de dólares) como parte de un crédito para apoyar el posconflicto (Deutsche Welle, 2017). Además, el Bun-
El rol de la Unión Europea como actor externo en la política colombiana - entre el bilateralismo y el trilateralismo

Klaus Bodemer 
destag celebró el 13 de enero de 2017 el lanzamiento oficial del Instituto Colombo Alemán para la Paz (Capaz) que tendrá sede en Bogotá. Se trata de un instituto que contará con la participación de un consorcio formado por cinco universidades alemanas y cinco colombianas bajo el liderazgo de la Universidad Nacional de Bogotá y la Universidad de Gießen. La idea básica de esta iniciativa es que Capaz aporte al proceso de paz con investigación, docencia y eventualmente la consultaría o extensión (El Espectador, 2017). El director del instituto debe ser un profesor alemán.

(12) Con respecto a la reforma al campo, un desafío adicional del posconflicto, Maihold y Naucke han propuesto que esta consista en dos componentes: primero, la distribución de tierras a pequeños agricultores que hasta ahora no las han tenido; segundo, la restitución de las cerca de seis millones de hectáreas a los casi seis millones de desplazados, como la Ley de Restitución de Tierras lo destaca. Eso no va a ser fácil, porque en muchas regiones que hasta ahora estaban en manos de la guerrilla no existe ni un catastro ni un registro de la propiedad y los tratados de compra se han arreglados hasta ahora con apretón de manos. Existe, además, la preocupación de que la concesión de títulos de campo podría ser el primer paso a su expropiación. Por eso se necesitarían garantías internacionales en este proceso. A fin de que el Estado colombiano disponga de los fondos necesarios para esto, es necesario, además, una reforma del sistema de impuestos, que es muy regresivo y complejo, contiene muchas excepciones y una base muy baja. También en esta área algunos países europeos, entre ellos Alemania, tienen mucha experiencia y podrían ofrecer apoyo técnico.

(13) Frente al hecho de que la economía colombiana está muy afectada por los bajos precios de materias primas, su política económica debería cambiar la marcha, hacerse más independiente de la exploración y exportación de materias primas e invertir más en una política industrial sostenible. También en esta área existe mucha experiencia en Europa. ${ }^{3}$ Tomando en cuenta una de las causas históricas del conflicto armado, la cuestión agraria, el fomento de los pequeños agricultores debería ser un elemento central de este cambio de rumbo, el cual ofrecería la oportunidad de mejor integración de algunas partes de los excombatientes (Maihold y Naucke, 2015, p. 6).

3 Por ejemplo, en Alemania con la Deutsche Gesellschaft für Internationale Zusammenarbeit, GIZ (Agencia Alemana de Cooperación Internacional) y el Deutsche Institut für Entwicklungspolitik, DIE (Instituto Alemán de Desarrollo). La GIZ dispone, junto con la Comisión Económica para América Latina y el Caribe, CEPAL, de mucha experiencia en América Latina con la aplicación del concepto de la competitividad sistémica. 


\section{Referencias bibliográficas}

Agencia Presidencial de Cooperación Internacional de Colombia (2015). Programa Regional de Cooperación con Mesoamérica. Recuperado de https://antiguoapccolombia. gov.co/?idcategoria $=100$

Ambos, K. (2016). Aval del parlamento alemán para proceso de paz. El Espectador, 12 de julio. Recuperado de https://www.elespectador.com/opinion/opinion/aval-delparlamento-aleman-para-proceso-de-paz-columna-642940

Arcos Molas, M. (2015). Colombia: un país sumido en medio siglo de conflicto. Recuperado de http://www.ieee.es/publicaciones-new/documentos-marco/2015/DIEEEM01-2015.html

Bancet, A. (2012). Análisis de la cooperación triangular: discursos y prácticas de los países del CAD/OCDE sobre una modalidad en construcción. Serie "Documentos de Trabajo". Recuperado de http: realidad-de-la-ayuda.s3.amazonaws.com/uploads/document/file/7/ An_lisis_cooperaci_n_triangular.pdf

Bodemer, K. (2000). Vom Andenpakt zur Andengemeinschaft. En S. Kurtenbach, K. Bodemer y D. Nolte (eds.), Sicherheitspolitik in Lateinamerika. Vom Konflikt zur Kooperation (pp. 4548). Opladen: Leske + Budrich.

Bodemer, K. (2015). Gemeinsamer Markt des Südens. Mercosur. En A. Grimmel y C. Jacobeit (eds.), Regionale Integration: Erklärungsansätze und Analysen zu den wichtigsten Integrationszusammenschlüssen in der Welt (Forschungsstand Politikwissenschaft) (pp. 373400). Baden-Baden: Nomos Verlagsgesellschaft.

Cala Campo, A. (2015). Colombia y la crisis petrolera. Madrid: Real Instituto Elcano, ARI 38-20/72015. Recuperado de http://www.realinstitutoelcano.org/

Cardona Cardona, D. (2011). Introducción. ¿Puede tener Colombia una estrategia de política exterior? En D. Cardona Cardona (Ed.), Colombia: una política exterior en transición. Bogotá: Friedrich Ebert Stiftung en Colombia- FESCOL, XV-XII.

Comisión Europea (2007b). Documento de Programación Regional-América Latina 20072013. Recuperado de https://ec.europa.eu/europeaid/documento-de-programaci \%C3 \%B3nregional- \%E2 \%80 \%93-am \%C3 \%A9rica-latina-2007-2013_es

Delegación de la Unión Europea en Colombia (s/f). Colombia y la Unión Europea. Recuperado de http://eeas.europa.eu/archives/delegations/colombia/eu_colombia/political_relations/index_es.htm

De Lombaerde, P. et al. (2006). EU Policies Towards the Colombian Conflict: Policy Coordination and Interregionalism. OBREAL / EULARO Background Paper, April. Recuperado de http://www10.iadb.org/intal/intalcdi/PE/2009/03383.pdf.

Doidge, M. (2011), The European Union and Interregionalism. Farnham: Ashgate.

Domínguez, R. (2013). El pragmatismo en las relaciones UE y Colombia/Perú. En J. Roy (comp.), Después de Santiago: integración regional y relaciones Unión Europea-América Latina (pp. 105-115). Miami, Florida: European Union Center.

Duarte García, J. (2012). La Unión Europea en la perspectiva de la política exterior de Colombia y Brasil: escenarios bilaterales e interregionales. En E. Pastrana Buelvas, S. Jost y D. Flemes (eds.), Colombia y Brasil: ¿socios estratégicos en la construcción de Suramérica? (pp. 339-365). Bogotá: Pontificia Universidad Javeriana.

Duarte García, J. (2014). La cooperación para el desarrollo de la Unión Europea en Suramérica: transformaciones y oportunidades. En E. Pastranas Buelvas y H. Gehring
El rol de la Unión Europea como actor externo en la política colombiana - entre el bilateralismo y el trilateralismo

Klaus Bodemer 
(eds.), Suramérica en el escenario global: gobernanza multinivel y birregionalismo (pp. 435461). Bogotá: Pontificia Universidad Javeriana.

Flemes, D. y Castro, R. (2015). Colombia y Brasil frente a la Alianza del Pacífico y el Mercosur. Iberoamericana, 15(60), pp. 200-204.

Flemes, D., Wehner, L. (2015). Drivers of Strategic Contestation: The Case of South America. International Politics, 52 (2), pp. 163-177.

Friedrich Ebert Stiftung en Colombia (2004). Relaciones Colombia-Unión Europea: De la incertidumbre politica al posicionamiento estratégico. FES- Policy Paper, 4, Bogotá.

Fundación Paz \& Reconciliación (2017). Terminó la guerra, el postconflicto está en riesgo. A un año del acuerdo de paz, 23 de noviembre, Bogotá. Recuperado de https://www.pares. com.co/wp-content/uploads/2017/11/TERMINO-LA-GUERRA-EL-POSTCONFLICTOESTA-EN-RIESGO-2.pdf

Grevi, G. (2010), Making EU Strategic Partnerships Effective, FRIDE, Working Paper 105, 20 p. Madrid.

Infolatam (21 diciembre 2015). CE concede tres millones a proyectos cooperación con América Latina y Caribe. Recuperado de http://www.infolatam.com/2015/12/21/ce-concede-tresmillones-a-proyectos/

Johnson, K. (2017). El papel de Europa en las negociaciones con las FARC. Razón Publica, 2 de octubre 2016. Recuperado de https://www.razonpublica.com/index.php/ conflicto-drogas-y-paz-temas-30/9749-el-papel-de-europa-en-las-negociaciones-conlas-farc.html

Keohane, R. (1984). After Hegemony: Cooperation and Discord in the World Political Economy. Princeton: Princeton University Press.

Kurtenbach, S. (2004), El papel de los actores externos en la contención de la violencia en Colombia. Hamburgo: Instituto de Estudios Iberoamericanos.

Loser, C. (2015), ¿Qué pasó con la década de América Latina? Recuperado de http://www. infolatam.com/2015/09/02(que-paso-con-la-decada-de-america-latina/

Maihold, G. y Naucke, P. (2015). Kolumbiens Weg zum Frieden: Die Verhandlungen zwischen der Regierung und den Fuerzas Armadas Revolucionarias de Colombia-Guerilla bedürfen internationaler Begleitung. SWP-Aktuell.

Malamud, C., García Ensina C. (2013). Colombia, la OTAN y las alarmas que suenan en América Latina, Comentario Elcano 40 18/6. Recuperado de http://www.realinstitutoelcano. org/

Manetto, F. (2017). La pugna por el control de la coca agrava el conflicto social en Colombia. El País, 10 de octubre. Recuperado de https://elpais.com/internacional/2017/10/08/ colombia/1507497356_961918.html

Morazán, P., Sanahuja, J.A. y Ayllón, B. (2011). Una nueva política de cooperación para el desarrollo de la Unión Europea con América Latina. Parlamento Europeo, Dirección General de Políticas Exteriores de la Unión, Dirección B, Departamento Temático, EXPO/B/DEVE/ FWC/2009-01/Lot5/18, diciembre.

Pacheco, K. (2002). Panorama de las relaciones entre la Unión Europea y la Comunidad Andina de Naciones. En C. Freres y K. Pacheco (eds.), Nuevos horizontes andinos: escenarios regionales y políticas de la Unión Europea (pp. 163-176). Caracas: Nueva Sociedad.

Pada, P. (2006). The Intermestic Affairs: A Theoretical Approach. Recuperado de http:// perrypada.blogspot.com.co/2006/04/intermestic-affairs-theoretical.html. 
Pastrana Buelvas, E. (2009). Las relaciones Colombia-Unión Europea: estancamiento o profundización. Revista Javeriana, 753, pp. 28-35.

Pastranas Buelvas, E. y Aponte Castro, D. (2006). La Unión Europea como potencia civil: la estrategia de los laboratorios de paz en Colombia. Revista Diálogos de Saberes, 25 , pp. 241-270.

Pastrana, E.,Vera Pineros, S. (2015). La seguridad y la defensa en las relaciones entre Brasil y Colombia: convergencias, obstáculos y distancias. Iberoamericana, 15(60), pp. 185-189.

Putnam, R.D. (1988). Diplomacy and Domestic Politics. The Logic of Two-Level Games. International Organizations Journal, vol. 42, no. 3, pp. 427-460.

Sanahuja, J. A. (2013). La Unión Europea y el regionalismo latinoamericano: un balance. Investigación y Desarrollo, 21(1), pp. 156-184.

Sanahuja, J.A., Tezanos Vásquez, S., Kern A. y Perrota, D. (2015). Más allá de 2015: perspectivas y propuestas para la cooperación al desarrollo entre la UE y América Latina y el Caribe. Hamburgo: Fundación Unión Europea-América Latina y el Caribe.

Sin autor (2017). UE ya aprobó programa de 11 millones de euros para el posconflicto. El País, 11 de julio. Recuperado de http://www.elpais.com.co/.../ue-ya-aprobo-programade-11-millones-de-euros-para-el-posc

Sotillo Lorenzo, J. Á. y Bendelac Gordon, L. (2013). Cooperación Sur-Sur y Triangulación en América Latina en la Unión Europea. Documento de Trabajo, 26. Recuperado de https:// www.ucm.es/data/cont/docs/599-2014-03-12-DT26_CSS_CTR_UEAL.pdf

Steinberg, F. (30 de junio 2014). América Latina ante el Acuerdo Transpacífico de Cooperación Económica y Tratado Transatlántico de Comercio e Inversiones. Comentario Elcano, 44. Recuperado de http://www.realinstitutoelcano.org/wps/portal/web/rielcano_ es/contenido?WCM_GLOBAL_CONTEXT=/elcano/elcano_es/zonas_es/comentariosteinberg-america-latina-ante-tpp-y-ttip

Waltz, K. (1979), Theory of International Politics. Boston: McGraw-Hill.

Welsch, F. y Briceño, H. (2011). Chávez und der Chavismo. En: A. Boeckh, F. Welsch y N. Werz (eds.), Venezuela heute: Politik-Wirtschaft-Kultur (pp. 107-130). Frankfurt a. M., Vervuert.

Wendt, A. (1999). Social Theory of International Politics. Cambridge: Cambridge University Press.

Zibechi, R. (2011). Brazil and Colombia: An Unexpected Alliance. Foreign Policy in Focus. Recuperado de http://fpif.org/brazil_and_colombia_an_unexpected_alliance/
El rol de la Unión Europea como actor externo en la política colombiana - entre el bilateralismo y el trilateralismo

Klaus Bodemer 